\section{Situação de Saúde do Idoso: Ensino -Pesquisa-Extensão em um Município do Centro-Oeste Paulista}

\author{
Health Condition of Elderly: Teaching-Research-Extension \\ in a Municipality Based in the Mid-West of São Paulo State
}

\section{RESUMO}

Poucos são os estudos nacionais acerca da saúde do idoso que privilegiam a avaliação geriátrica multidimensional e a qualidade de vida por meio de instrumentos de mensuração. Esta pesquisa busca relatar uma experiência interdisciplinar de aplicação de instrumentos de avaliação do perfil socioeconômico, da qualidade de vida, da função cognitiva e da presença/gravidade do zumbido de idosos participantes de uma atividade de ensino, pesquisa e extensão, em um município do centro-oeste paulista. Pesquisa de caráter empírico, descritivo e transversal. Para coleta de dados foram utilizados: Mini Exame do Estado Mental; Critério de Classificação Econômica Brasil; Questionário de Qualidade de Vida SF-36 e Questionário de Gravidade do Zumbido e para análise: Testes de Mann-Whitney e Correlação de Spearman. Resultados: escores elevados em relação ao estado geral de saúde e aspectos sociais e mais baixos para limitação por aspectos funcionais e gravidade do zumbido. A análise revelou que o estado de saúde de idosos participantes da feira está intrinsecamente relacionado às condições de vida e trabalho da população. Os dados encontrados podem subsidiar políticas públicas e ações de promoção da saúde, pois estão alicerçados no entendimento de que a situação de saúde dos idosos é resultado da interação de múltiplos fatores.

Pallaviras-chave: Idoso. Qualidade de Vida. Promoção da Saúde. Extensão Comunitária.

\section{ABSTRACT}

There are few national studies about elderly people health, which highlight the multidimensional geriatric evaluation and the quality of life through measuring instruments. This study aims report an interdisciplinary experience of applying instruments for assessing the socioeconomic profile, quality of life, cognitive function, and the presence/severity tinnitus of elderly participants in a learning activity, research in a

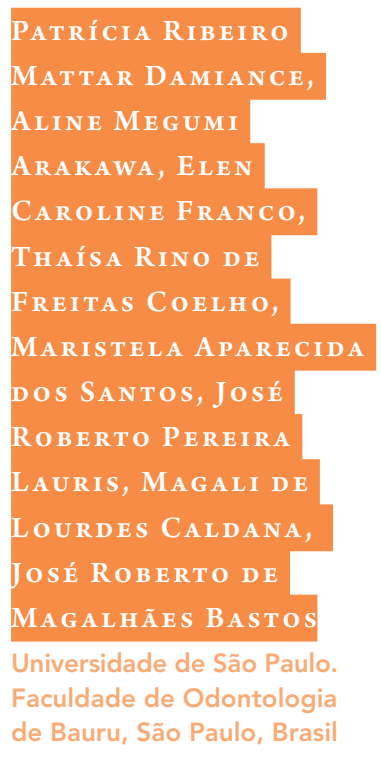

Patrícia Ribeiro

Mattar Damiance,

Aline Megumi

Arakawa, Elen

Caroline Franco,

THAísa Rino de

Freitas Coelho,

Maristela Aparecida

Dos Santos, José

Roberto Pereira

Lauris, Magali de

LOURdes Caldana

JOSÉ ROBERTO DE

MAGALHÃES BASTO

de Bauru, São Paulo, Brasi 
municipality based in the mid-west state of São Paulo. Empiric, descriptive and transversal research. For data collection the following questionnaires were employed: Mini Exam of Mental Status; Brazil Economic Classification Criteria; Quality of Life Questionnaire SF-36 and Tinnitus Handicap Inventory, and for analysis the following tests were employed: Mann-Whitney Test and Spearman Correlation Test. The results showed: high scores concerning general health and social aspects, and lower scores for limitation due to functional aspects and for tinnitus severity. Analysis showed that the health of the elderly who participated in the event is intrinsically related to the conditions of population's life and work of the population. Data may support public policies and actions to promote health because they are based on the comprehension that health condition of elderly people results on the interaction of multiple factors.

Keywords: Aged. Quality of Life. Health Promotion. Community-institutional Relations.

\section{INTRODUÇÃO}

\section{O envelhecimento populacional, com todas as suas peculiaridades sociais,} políticas, econômicas, biológicas e psicológicas, é um desafio principalmente para os países em desenvolvimento. A elaboração de políticas públicas e de saúde que atendam todas as demandas do processo de envelhecimento, considerando os determinantes sociais da saúde e as especificidades anatômicas e fisiológicas do envelhecimento, sob a ótica da integração ensino-serviço, é de extrema relevância para promover saúde e Qualidade de Vida (QV) a essa população.

O artigo $7^{\circ}$ da Lei 8080 de 1990, que trata dos princípios do Sistema Único de Saúde (SUS), preconiza que as ações e serviços públicos e privados que integram o sistema de saúde devem utilizar a epidemiologia para o estabelecimento de prioridades, alocação de recursos e orientação programática; e o artigo $14^{\circ}$ salienta a necessidade da criação de Comissões Permanentes de Integração entre os serviços de saúde e as instituições de ensino profissional e superior, com a finalidade de propiciar a formação acadêmica e educação continuada de profissionais para o SUS, assim como pesquisas e cooperação técnica, considerando as atribuições de cada esfera de governo [6]. Nesse contexto, a parceria entre a Academia e os gestores de saúde, em atividades de estágio, pesquisa, extensão e educação em saúde, pode adequar a formação às necessidades do SUS, propiciar levantamentos de necessidades individuais e coletivas de saúde, traçar o perfil de saúde do município e desenvolver políticas que visem aumentar a cobertura e a qualidade das ações em saúde [13].

A Política Nacional de Saúde da Pessoa Idosa [8], em suas diretrizes, apoia o desenvolvimento de estudos e pesquisas de avaliação da qualidade da atenção à saúde do idoso e seu aprimoramento. Além disso, estimula a formação de redes de apoio com as instituições formadoras como: universidades, centros universitários, faculdades e órgãos públicos a fim de fomentar, apoiar e identificar pesquisas em envelhecimento e saúde do idoso pensando na socialização, divulgação, financiamento e embasamento de novas investigações. Destaca a relevância científica, social e política de se investigar as condições que interferem no bem-estar na senescência e os fatores associados 
à $\mathrm{QV}$, no intuito de criar alternativas de intervenção e propor ações e políticas na área da saúde, buscando atender as demandas (biológicas, psicossociais, funcionais, financeiras) da população que envelhece.

Dessa forma, a avaliação do estado de saúde do idoso está diretamente relacionada à $\mathrm{QV}$, influenciada pelo sexo, escolaridade, idade, condição econômica e presença de incapacidades [26]. A avaliação integral da pessoa idosa, em uma perspectiva multidimensional, que considera aspectos de ordem biológica, psíquica e social é a maneira mais adequada de mensurar a saúde do idoso e planejar ações de saúde condizentes com as necessidades dessa população [30].

Em relação às necessidades específicas da população idosa, vale lembrar que o idoso sofre alterações decorrentes do próprio processo de envelhecimento, modificando muitas funções de órgãos e sistemas, principalmente do Sistema Nervoso Central (SNC) e os órgãos dos sentidos, especialmente, a audição. Um dos problemas associados à perda auditiva é o zumbido que influencia profundamente no desenvolvimento psicossocial do indivíduo senil, ocasionando dificuldades de integração, socialização, autoimagem e autoestima $[15,16]$. O zumbido severo é considerado o terceiro pior sintoma que pode acometer o ser humano, perdendo somente para a dor crônica e a tontura intensa intratável. Em aproximadamente $80 \%$ dos casos, o zumbido é leve e intermitente, o que não afeta em praticamente nada a vida diária do indivíduo, nem mesmo o leva a procurar ajuda médica [5]. Entretanto, no indivíduo senil o impacto do zumbido na QV precisa ser mensurado [17].

Poucos são os estudos nacionais acerca da saúde do idoso que privilegiam a avaliação geriátrica multidimensional e a QV por meio de instrumentos de mensuração. Em uma revisão sistemática [25] de estudos sobre a QV indexados na base de dados da Scientific Electronic Library Online (SciELO), realizada no período de janeiro de 2001 a dezembro de 2006, com o objetivo de analisar a produção científica sobre o tema, foram encontrados 217 artigos utilizando o descritor "qualidade de vida" e um artigo com o descritor "qualidade de vida relacionada à saúde". Por ano de publicação, levantaram-se 180 artigos. Destes, 124 possuíam o assunto QV como objeto central. Entre alguns dos resultados, os autores verificaram que a produção científica sobre a QV relacionada à saúde do idoso representou somente $5,8 \%$ dos estudos e que o idoso não foi foco temático de pesquisas que utilizaram instrumentos de medida da QV e nem na pesquisa qualitativa.

Conforme exposto, este estudo tem por objetivo relatar uma experiência interdisciplinar de aplicação de instrumentos de avaliação do perfil socioeconômico, da qualidade de vida, da função cognitiva e da presença/gravidade do zumbido de idosos participantes de uma atividade de ensino, pesquisa e extensão, em um município do centro-oeste paulista.

\section{MÉTODOS}

Estudo empírico, quantitativo, desenvolvido em parceria entre o Departamento de Saúde Coletiva da Faculdade de Odontologia de Bauru da Universidade de São Paulo 
(FOB-USP), campus de Bauru e a Prefeitura Municipal de Agudos representada pela Secretaria Municipal de Higiene e Saúde (SMHS). As instituições promoveram a Feira da Melhor Idade, entre os dias 11 e 14 de junho de 2012, no período vespertino, na praça central da cidade.

A feira fundamentou-se em atividades de atenção à saúde, extensão, educação e pesquisa, direcionadas à população idosa do município, com um enfoque interdisciplinar e visão multidimensional da assistência à saúde do idoso, envolvendo profissionais da rede municipal de saúde e estudantes da pós-graduação de diversas áreas do conhecimento, tais como: saúde, ciências sociais e humanas. As atividades de atenção à saúde concentraram-se na oferta da vacinação contra a Influenza A; verificação de pressão arterial e glicemia capilar; exame clínico bucal, aprendizado sobre técnicas de autoexame bucal, inspeção do meato acústico externo (orelha) e cuidados para manter a integridade da audição.

As atividades de educação em saúde foram realizadas individualmente, conforme as necessidades percebidas na interação, e coletivamente, por meio de impressos sobre: Doença de Parkinson e Alzheimer, Acidente Vascular Encefálico, presbifonia (processo natural de envelhecimento vocal), presbiacusia (processo natural de envelhecimento auditivo) e presbifagia (processo natural de envelhecimento das estruturas musculares da mastigação e deglutição).

Os indivíduos com 60 anos ou mais de idade foram convidados a participar de pesquisas relacionadas a diversos aspectos da saúde do idoso, respondendo questionários validados na literatura. Todos os que aceitaram participar receberam explicação sobre os aspectos éticos e assinaram os Termos de Consentimento Livre e Esclarecido, apreciado pelo Comitê de Ética em Pesquisa da FOB-USP e aprovados sob o protocolo no 02978212.8.00o0.5417, conforme a resolução n. 196/96 do Conselho Nacional de Saúde.

Dentre aqueles, foram selecionados 44 idosos, independentemente do sexo, submetidos ao Mini-Exame do Estado Mental (MEEM) [11] e ao Critério de Classificação Econômica Brasil (CCEB) [1], ao Questionário de Qualidade de Vida Medical Outcomes Study 36-Item Short-Form Health Survey (SF-36) [12] e a um Questionário de Gravidade do Zumbido (QGZ) [16]. Estes instrumentos foram apreendidos pelos pós-graduandos, nas etapas preparatórias ao evento.

Para o Questionário Socioeconômico foi utilizado o Critério de Classificação Econômica Brasil (CCEB), proposto pela Associação Brasileira de Empresas de Pesquisa (ABEP) [1]. Nesta escala, a escolaridade do chefe da família vale de o a 8 pontos; os demais pontos são fornecidos pela quantidade de bens de consumo duráveis que a família possui (automóvel, televisão em cores, rádio, geladeira, freezer, máquina de lavar roupa etc.), a quantidade de cômodos da casa, enfatizando-se os banheiros e quantidade de empregados mensalistas trabalhando para a família. $\mathrm{O}$ questionário define a classe econômica dos indivíduos avaliados, através de escores que vão de o-7 pontos para a classe E até 42-46 pontos para a classe A1.

O SF-36 foi elaborado para avaliação da QV. No Brasil, o instrumento se mostrou adequado às condições socioeconômicas e culturais da população. Composto por 11 
questões e 36 itens que envolvem oito domínios, sendo representados por capacidade funcional (dez itens), aspectos físicos (quatro itens), dor (dois itens), estado geral da saúde (cinco itens), vitalidade (quatro itens), aspectos sociais (dois itens), aspectos emocionais (três itens), saúde mental (cinco itens) e uma questão comparativa sobre a percepção atual da saúde. $\mathrm{O}$ indivíduo recebe um escore em cada domínio, que varia de zero a $100[12]$.

O MEEM foi criado e validado por Folstein, Fostein e McHugh, em 1975, com o objetivo de avaliar a função cognitiva e identificar os indivíduos que possuem alterações das funções cognitivas. Pode ser utilizado em avaliações iniciais e seriais do funcionamento mental, pois demonstra quantitativamente a piora ou melhora da função mental de acordo com o tempo e possíveis tratamentos. Apresenta também capacidade de avaliar questões relacionadas ao autocuidado. $\mathrm{O}$ exame consiste em respostas verbais para questões sobre orientação tempo-espacial, memória, atenção e cálculo, cuja possibilidade de pontuação máxima é 21 pontos. Já a capacidade do indivíduo denominar, seguir ordens verbais e escritas, escrever uma sentença de forma espontânea e desenhar por meio de cópia dois polígonos em intersecção (respostas escritas), com a possibilidade máxima de pontuação de nove pontos. A parte verbal e a escrita totalizam o máximo de 30 pontos. $[4,11]$

Em nosso meio, pesquisadores [4] indicaram a necessidade de adequar a pontuação inicialmente proposta, considerando o nível de escolaridade da população brasileira. Os valores propostos foram: 13 pontos para os analfabetos, 18 para as pessoas com escolaridade baixa e média e 26 para escolaridade alta. Outra adaptação do teste foi proposta [11], para uso em ambiente hospitalar, consultório e estudos populacionais modificando palavras, no item orientação tempo-espacial, e fornecendo regras para aplicação uniforme do instrumento. A pontuação e a estrutura do instrumento, no quesito orientação espacial, obedeceram, respectivamente, aos dois estudos.

O QGZ foi escolhido por ser considerado o mais completo na avaliação dos aspectos psicológicos e cotidianos do zumbido [16]. Além disso, possui uma boa confiabilidade, ratificada por altas consistências internas. A facilidade, a rapidez na aplicação (cerca de cinco minutos) e a reprodutibilidade (não apresenta reserva de direitos autorais) são outras justificativas para a escolha do teste. Composto por 25 questões, divididas em três escalas. A funcional (F) mede o incômodo provocado pelo zumbido em funções mentais, sociais, ocupacionais e físicas. A escala emocional (E) mede as respostas afetivas como ansiedade, raiva e depressão. A catastrófica (C) quantifica o desespero e a incapacidade referida pelo acometido para conviver ou livrar-se do sintoma. São três as opções de resposta para cada uma das questões, pontuadas da seguinte maneira: para as respostas "sim" (4 pontos), às vezes ( 2 pontos) e "não" (nenhum ponto). As respostas são pontuadas de zero, quando o zumbido não interfere na vida do paciente, até 100 (pontos ou \%), quando o grau de incômodo é grave. A somatória dos pontos resultantes das questões é categorizada em cinco grupos ou graus de gravidade e o zumbido pode ser: desprezível (o-16\%), leve (18-36\%), moderado (38-56\%), severo (58-76\%) ou catastrófico (78-100\%).

Os dados coletados foram registrados, tabulados e receberam tratamento estatístico: Teste Mann-Whitney e Teste de Correlação de Spearman. 


\section{RESULTADOS}

Os resultados das variáveis sociodemográficas e da aplicação dos instrumentos estão descritas na Tabela 1. A faixa etária dos idosos variou entre 60 e 85 anos de idade, sendo 6,0 o desvio padrão, 67,86 anos a média etária e 67 anos a mediana. Com relação à escolaridade e classe social houve prevalência de idosos analfabetos e pertencentes à classe $\mathrm{B}_{2}$, respectivamente.

Tabela 1 - Caracterização sociodemográfica da amostra

\begin{tabular}{|c|c|c|c|}
\hline & & $\mathrm{N}$ & $\%$ \\
\hline \multirow{2}{*}{ GÊNERO } & Feminino & 25 & 56,81 \\
\hline & Masculino & 19 & 43,18 \\
\hline \multirow{6}{*}{ ESCOLARIDADE } & Analfabeto & 18 & 40,90 \\
\hline & Fundamental incompleto & 16 & 36,36 \\
\hline & Fundamental completo & 3 & 6,81 \\
\hline & Médio incompleto & 0 & 0 \\
\hline & Médio completo & 5 & 11,36 \\
\hline & Superior completo & 2 & 4,54 \\
\hline \multirow{5}{*}{ CLASSE SOCIAL } & B1 & 4 & 9,09 \\
\hline & B2 & 13 & 29,54 \\
\hline & $\mathrm{C} 1$ & 8 & 18,18 \\
\hline & $\mathrm{C} 2$ & 10 & 22,72 \\
\hline & $D$ & 9 & 20,45 \\
\hline
\end{tabular}

No que se refere à QV, os indivíduos apresentaram escores elevados nos domínios que avaliaram o Estado Geral de Saúde $(80,32)$, Aspectos Sociais $(82,10)$ e Limitação por Aspectos Emocionais $(82,58)$. Os escores mais baixos foram encontrados nos domínios Dor $(73,73)$ e Limitações por Aspectos Funcionais $(71,59)$. Em cada domínio do SF-36, as mulheres obtiveram escores menores do que os homens, exceto nos domínios EGS e V, porém não foram encontradas diferenças estatisticamente significativas (Tabela 2). 
Tabela 2 - Escores do questionário SF-36 de acordo com os domínios avaliados

\begin{tabular}{|c|c|c|c|c|c|c|c|c|}
\hline \multirow[b]{2}{*}{ DOMÍNIOS } & \multicolumn{6}{|c|}{ GERAL } & \multicolumn{2}{|c|}{ GÊNERO } \\
\hline & $x$ & $\mathrm{DP}$ & MíN & MÁX & MED & ESCORE MÁX & FEMININO & MASCULINO \\
\hline $\mathrm{CF}$ & 69,77 & 26,74 & 0 & 100 & 70 & 100 & 66 & 74,74 \\
\hline LAF & 71,59 & 34,35 & 0 & 100 & 75 & 100 & 68 & 76,32 \\
\hline D & 73,73 & 18,47 & 41 & 100 & 72 & 100 & 72,76 & 75,00 \\
\hline EGS & 80,32 & 17,22 & 17 & 100 & 83,50 & 100 & 81,92 & 78,21 \\
\hline V & 74,32 & 19,99 & 25 & 100 & 80 & 100 & 75,80 & 72,37 \\
\hline AS & 82,10 & 23,56 & 25 & 100 & 100 & 100 & 81 & 83,44 \\
\hline LAE & 82,58 & 36,29 & 0 & 100 & 100 & 100 & 8133 & 84,21 \\
\hline SM & 76,36 & 19,34 & 28 & 100 & 84 & 100 & 80,32 & 71,16 \\
\hline
\end{tabular}

CF: Capacidade Funcional; LAF: Limitação por Aspectos Físicos; D: Dor; EGS: Estado Geral de Saúde; V: Vitalidade; AS: Aspectos Sociais; LAE: Limitação por Aspectos Emocionais; SM: Saúde Mental; x: média; dp: desvio padrão; Mín: mínimo; Máx: máximo; Med: mediana.

Ao realizar a comparação entre a $\mathrm{QV}$ e o sexo dos participantes, não foram encontradas diferenças estatisticamente significativas. Correlacionando-se a $\mathrm{QV}$ com a classificação socioeconômica, escolaridade e pontuação no MEEM (Tabela 3), pode-se observar uma análise positiva linear com diferença estatisticamente significativa nos domínios CF, LAE e LAF. Em outras palavras, lê-se que quanto maior a qualidade de vida, melhor a CF e menores LAE e LAF. Salienta-se que quanto maior a escolaridade, maiores são as pontuações no MEEM, que, por sua vez, apresentou pontuação máxima de 30 pontos, mínimo de 13 pontos, 4,13 de desvio padrão, média de 23,64 pontos e mediana de 24 pontos. 
Tabela 3 - Correlação entre a qualidade de vida, classificação socioeconômica, escolaridade e aspectos cognitivos.

\begin{tabular}{|c|c|c|c|c|c|c|}
\hline \multirow[b]{2}{*}{ DOMÍNIOS } & \multicolumn{2}{|c|}{$\begin{array}{l}\text { CLASSIFICAÇÃO } \\
\text { SOCIOECONOOMICA }\end{array}$} & \multicolumn{2}{|c|}{ ESCOLARIDADE } & \multicolumn{2}{|c|}{ ASPECTOS COGNITIVOS } \\
\hline & $\mathrm{R}$ & $P$ & $\mathrm{R}$ & $P$ & $\mathrm{R}$ & $P$ \\
\hline CF & 0,43 & $0,00^{\star}$ & 0,34 & $0,02^{\star}$ & 0,04 & 0,79 \\
\hline LAF & 0,25 & 0,09 & 0,30 & $0,04^{\star}$ & 0,31 & $0,04^{*}$ \\
\hline D & 0,10 & 0,50 & $-0,07$ & 0,64 & 0,02 & 0,91 \\
\hline EGS & 0,09 & 0,55 & $-0,14$ & 0,36 & $-0,22$ & 0,15 \\
\hline V & 0,24 & 0,12 & 0,04 & 0,78 & 0,07 & 0,65 \\
\hline AS & 0,09 & 0,57 & 0,02 & 0,87 & 0,12 & 0,45 \\
\hline LAE & 0,38 & $0,01^{*}$ & 0,29 & 0,05 & 0,35 & $0,02^{\star}$ \\
\hline SM & 0,15 & 0,34 & $-0,09$ & 0,57 & $-0,06$ & 0,70 \\
\hline
\end{tabular}

CF: Capacidade Funcional; LAF: Limitação por Aspectos Físicos; D: Dor; EGS: Estado Geral de Saúde; V: Vitalidade; AS: Aspectos Sociais; LAE: Limitação por Aspectos Emocionais; SM: Saúde Mental.

Dentre os 44 participantes, 20 (45,45\%) confirmaram a presença do zumbido e em meio a esta amostra, verificou-se que $55 \%$ dos participantes obtiveram o grau "desprezível” de severidade do zumbido, 25\% grau leve, $20 \%$ grau moderado e $\% \%$ para grau severo e catastrófico. Foi realizada correlação dos dados entre $Q V$ e zumbido não se encontrando dados estatisticamente significativos.

\section{DISCUSSÃO}

Com todas as ressalvas em relação ao número de participantes, homens e mulheres buscaram, de maneira igualitária, a atividade de extensão, utilizando como base para o raciocínio o percentual de homens e mulheres com mais de 60 anos, no município de Agudos-SP, que é respectivamente, 44,32\% e 55,68\% [20].

Os analfabetos e analfabetos funcionais representaram $77,26 \%$ e os indivíduos com mais de quatro anos de instrução, 22,72\%. O termo analfabeto funcional aplica-se aos indivíduos que não concluíram o primeiro segmento do ensino fundamental. A taxa de analfabetismo funcional, em 2007 , era de $21,7 \%$, com tendência declinante. O Brasil apresenta uma taxa de analfabetismo residual de $2,4 \%$ entre a população jovem ( 15 a 24 anos de idade). Entre o grupo de 40 a 59 anos ainda é expressivo o percentual de analfabetismo: $36,5 \%$ e entre os maiores de $60,40,1 \%$. O analfabetismo, no Brasil, guarda relação com região e seu desenvolvimento socioeconômico, com a área (urbana ou rural) e com o gênero [22].

Os déficits educacionais da população idosa e de uma parcela significativa de jovens e adultos representam (e representarão) um desafio para a saúde pública no

* diferença estatisticamente significante 
quesito ações de educação em saúde, pois serão necessárias estratégias criativas (e adequadas didaticamente) para mudar comportamentos e estilos de vida que tem grande impacto na ocorrência de doenças crônicas [19]. Acrescenta-se à afirmação a necessidade de adequação dos instrumentos de avaliação das condições de saúde, principalmente os relacionados à avaliação da função cognitiva, as especificidades decorrentes da privação de estímulos físicos e sociais propiciados pela educação, tão importantes para o desenvolvimento cognitivo.

$\mathrm{O}$ maior percentual de indivíduos da amostra pertence às classes $\mathrm{B}$ e C. A maioria da população, no Brasil, pertence à classe $\mathrm{C}$, que mais cresce, devido aos investimentos na redução das desigualdades sociais e ao crescimento econômico, principalmente do emprego formal e da renda $[2,9]$. Nota-se a importância dos indicadores sociodemográficos e da atual estrutura da pirâmide etária brasileira (que apresenta uma relação positiva entre a população economicamente ativa e a população de dependentes) sobre o desenvolvimento econômico e competitividade da economia brasileira para as próximas décadas.

Encontrou-se uma associação positiva entre a QV e o nível socioeconômico, o que corrobora os achados de outros estudos $[28,35]$. Na análise dos oitos domínios do SF-36, os valores se apresentaram acima do valor 50, que é uma pontuação moderada. Mesmo não havendo diferenças estatisticamente significativas entre qualidade de vida e sexo, ao analisar a média dos domínios, as mulheres apresentaram menores pontuações nos domínios da qualidade de vida. Esta informação é similar à trazida por uma pesquisa na qual foi utilizada a mesma ferramenta para verificar a $Q V$ de indivíduos que sofreram AVE [24]. Em outro estudo os autores observaram uma prevalência maior dos escores no sexo masculino exceto para estado geral de saúde e aspectos emocionais [31].

As mulheres apresentaram pontuações mais elevadas no EGS e V contradizendo os achados da Pesquisa Nacional de Amostras de Domicílios de 2003 [21], em que as idosas até 80 anos declararam um estado de saúde pior do que os homens da mesma idade. Após os 80 anos, os homens declararam estado de saúde pior do que as mulheres. Entretanto, as idosas, independentemente da percepção de seu estado de saúde, procuram atendimento médico em maior proporção do que os idosos.

No MEEM as pontuações obtidas pelos participantes foram superiores ao esperado, considerando a escolaridade do grupo. Os idosos da pesquisa apresentaram desempenho adequado nas questões sobre orientação tempo-espacial, memória imediata, capacidade de denominar objetos, seguir ordens verbais e desenhar por meio de cópia dois polígonos em intersecção (resposta escrita). Nota-se um prejuízo, em todas as faixas etárias, em relação à memória de evocação, mais evidente na faixa etária de 71 a 80 anos e mais de 80 anos e entre os analfabetos e analfabetos funcionais. Estes não conseguiram pontuar no item relacionado à memória de evocação. Entre os analfabetos é importante destacar que a privação educacional reflete no desenvolvimento da linguagem, da abstração, da capacidade de conservar quantidades, do pensamento reversível e raciocínio hipotético-dedutivo. Assim, como possuem limitações para abstrair e realizar operações mentais, eles precisam ver, pegar e sentir para 
compreender. Seu pensamento está preso às limitações da realidade concreta [27].

Considerando o exposto, propõe-se refletir sobre a estrutura e a aplicabilidade do MEEM aos diferentes níveis de instrução e as limitações cognitivas. Apresentar verbalmente palavras e testar a memória imediata e logo em seguida mostrar os objetos relativos às palavras e testar a memória de evocação, talvez possa causar impacto positivo na pontuação de indivíduos com menos de quatro anos de instrução.

Com o envelhecimento ocorre uma diminuição de neurônios principalmente no córtex cerebral e esta diminuição pode exercer um papel relevante no declínio da memória e das funções cognitivas. Outro fator importante, para compreender o declínio das funções mentais durante a senescência é a presença no cérebro do idoso, mais precisamente no giro do hipocampo, área associada com a memória recente, de placas neuríticas (massas de proteína amilóide situadas entre os neurônios) e de emaranhados neurofibrilares (massas de neurofibrilas no interior do citoplasma neuronal), que em número pequeno parece não alterar as funções celulares. Sabe-se que indivíduos com Alzheimer e Parkinson possuem grande número de placas e emaranhados [30, 34]. Entretanto, verifica-se que as Doenças do SNC não têm impacto significativo nas principais causas elencadas de internações hospitalares de idosos no SUS e causas de mortalidade de idosos, já que ocupam a $10^{\mathrm{a}}$ posição [21].

É necessário lembrar que para análisar de maneira segura o estado mental atual (estado cognitivo) torna-se importante realizar uma cuidadosa observação da apresentação geral do indivíduo e da história pregressa do nível funcional [15]. A capacidade funcional pode ser avaliada através de depoimentos indicativos de dificuldades ou não, nos cuidados pessoais e nas atividades instrumentais (uso do telefone, transporte, compras, preparação de alimentos, tarefas domésticas, medicação, dinheiro), que podem limitar a independência do idoso na comunidade [15, 30].

Fatores importantes justificaram as pontuações dos participantes no MEEM, tais como: boa aparência; vestimenta adequada, conforme a estação do ano; linguagem, postura e comportamento adequados à idade e a clareza de ideias dos idosos, em relação ao seu passado educacional e suas limitações físicas. Isso revela o quanto é importante na avaliação cognitiva considerar o estado físico, social e a autonomia funcional e não só a pontuação do MEEM de acordo com a escolaridade e a sensibilidade do teste para decteção da demência.

A perda auditiva é um fator desencadeante do zumbido uma vez que danos ou degenerações do sistema auditivo podem ser geradores do zumbido, podendo provocar interferência na concentração e no equilíbrio emocional do indivíduo. Outras causas podem ser atribuídas ao zumbido como doenças otológicas, alterações cardiovasculares, doenças metabólicas, neurológicas, psiquiátricas, fatores odontológicos e possivelmente a ingestão de drogas, cafeína, nicotina e álcool, portanto, estes fatores podem incidir de forma associada $[23,29,33]$.

Não foi verificada correlação entre a gravidade do zumbido, o sexo e a QV, de acordo com o Teste de Mann-Whitney e Teste de Correlação de Spearman. Admite-se a interferência do zumbido na qualidade de vida, mas a tolerabilidade depende não só das características específicas do zumbido (sensação de frequência, sensação de intensidade, tipo de zumbido, entre outras), mas também do estado afetivo, emocional 
e da função mental do indivíduo que o apresenta [3]. Cabe aqui uma ressalva: a presença do zumbido independentemente do grau de severidade merece atenção do sistema de saúde, pois pode ser um indicativo de perda auditiva, gerando impacto na qualidade de vida do indivíduo.

Finalizando a discussão, é importante salientar que, os idosos que compuseram a amostra estão inseridos em um município com um pouco mais de 35 mil habitantes; com um parque industrial denso; com uma cobertura populacional estimada pelas equipes básicas de saúde de quase $100 \%$ e com um razoável acesso a média e alta complexidade $[7,20]$. Acredita-se que esses fatos, associados à participação em grupos de terceira idade, tenham sido determinantes para os resultados obtidos, uma vez que, a prática de atividades físicas, manuais e convivência social estimulante diminuem a ansiedade, elevam a autoestima e proporcionam bem-estar impactando na QV e até evitando que o zumbido tenha repercussão negativa em suas atividades de vida diária.

Outro ponto importante, diz respeito à SMHS do município, que tem se mostrado receptiva à integração ensino-serviço, autorizando e auxiliando atividades de pesquisa em seus serviços de saúde, pelos pós-graduandos do Programa de Ciências Odontológicas Aplicadas. A Feira da Melhor Idade foi uma ação inédita, entre a SMHS e o Programa de Pós-graduação, com enfoque interdisciplinar e visão multidimensional da atenção à saúde do idoso.

Esperava-se maior adesão dos idosos à Feira e principalmente nas atividades de pesquisa. Acredita-se que algumas situações possam ter interferido na adesão dos idosos, por exemplo, a logística selecionada pelos pós-graduandos, no planejamento da feira e principalmente na coleta de dados para pesquisas. Entretanto, uma situação em particular chamou a atenção e propiciou reflexões entre os pós-graduandos, no quesito adesão. Alguns idosos que se aproximaram da equipe da Feira tinham a expectativa de verificar a pressão arterial e glicemia capilar, porém essas ações não foram oferecidas em todos os dias do evento. Os idosos não expressaram interesse na avaliação da cavidade bucal e na inspeção do meato acústico externo, mesmo após diálogo com os pós-graduandos, relatando que não tinham dentes ou que não ouviam bem devido a idade, por conseguinte, não tinham problemas.

Esse fato tem relação com a percepção do estado de saúde pelo idoso que identifica suas necessidades e as classifica, ou seja, se o idoso, ao perceber seu estado de saúde, classificá-lo como ruim isso acarreta uma demanda, de qualquer natureza, aos serviços de saúde. Dessa forma, se os idosos, ao avaliarem sua saúde, acreditarem que precisam de atendimento médico ou atendimento de saúde especializado, e não de promoção da saúde e prevenção de agravos, eles não irão aderir a programas preventivos e educacionais e muito menos a ações pontuais [21,22].

A percepção do estado de saúde tem relação direta com o gênero e a idade, que determinam o comportamento em relação à busca por atendimento. As mulheres apresentam maior fidelização a programas preventivos e educacionais do que os homens e buscam mais os serviços da Atenção Básica [18]. A Política Nacional de Atenção Integral à Saúde do Homem discute a importância de se compreender as barreiras socioculturais e institucionais para traçar estratégias de promoção do acesso dos 
homens aos serviços de atenção primária, principalmente entre a população idosa e entre os idosos mais velhos [10].

Salienta-se que a parceria foi relevante para os pós-graduandos, que vivenciaram a integração ensino-serviço-extensão, trocaram conhecimentos e tecnologias com a sociedade e desenvolveram pesquisas sobre o envelhecimento, no campo de ação dos Determinantes Sociais da Saúde; para a população, que também trocou conhecimentos e tecnologias com os pós-graduandos, e de alguma maneira apreendeu informações e conhecimentos para prevenir ou procurar tratamento adequado para determinado problema de saúde, assim como para o gestor público municipal que terá em mãos informações e evidências para subsidiar políticas públicas, ações de promoção da saúde e de prevenção de doenças, fundamentadas na compreensão de que a situação de saúde dos idosos resulta da interação de múltiplos fatores.

\section{CONCLUSÃO}

Cientes das limitações da pesquisa, sem fazer generalizações, pode-se dizer que a análise revelou que o estado de saúde de idosos participantes da feira está intrinsecamente relacionado às condições de vida e trabalho da população. Ressalta-se nessa análise limitações como o tipo de pesquisa; o número de sujeitos envolvidos e a inexistência de outros tipos de estudos com o mesmo referencial teórico e metodológico.

A maior contribuição do estudo relaciona-se a mensuração com instrumentos de caráter multidimensional da QV relacionada à saúde, por avaliadores de diversas áreas do conhecimento, na tentativa de apreender a percepção do indivíduo sobre seu estado de saúde e funcionalidade na perspectiva da interdisciplinaridade e da abordagem integral da saúde da pessoa idosa. Os instrumentos de coleta de dados selecionados abrangeram domínios do estado cognitivo, biológico e socioeconômico tão necessário (e muitas vezes negligenciados) na avaliação do estado de saúde do idoso, na prescrição de cuidados, no delineamento de ações de promoção da saúde e prevenção de doenças e agravos e em estudos de efetividade dessas ações.

\section{REFERÊNCIAS}

[1] ABEP. Associação Brasileira de Empresas de Pesquisa. Critério de classificação econômica Brasil, 2010. Disponível em: <http://www.abep.org/novo/ Default.aspx>. Acesso em: 12 fev. 2014.

[2] AKERMAN, M. et al. Saúde e desenvolvimento: que conexões? In: Campos, G.W.S. et al. Tratado de Saúde Coletiva. São Paulo: Hucitec; 2007. p. 111-35.

[3] ALMEIDA, L.D. et al. Vestibulometria em indivíduos com zumbido e exames audiológicos normais. Rev CEFAC. São Paulo, v.7, n.3, p.382-387, mai. 2005.

[4] BERTOLUCCI, P.H. et al. O mini-exame do estado mental em uma população geral: impacto da escolaridade. Arq. Neuropsiquiatr. São Paulo, v. 52, n.1, p. 1-7, mar. 1994. DOI: http://dx.doi.org/10.1590/Sooo4-282X1994000100001

[5] BOGER, M. E. A influência do espectro de ruído na prevalência de perda auditiva induzida por ruído e zumbido em trabalhadores. 2007. $72 \mathrm{f}$. 
Dissertação (Mestre em Ciências da Saúde) - Universidade de Brasília, Brasília, 2007.

[6] BRASIL. Lei n. 8.080, de 19 de setembro de 1990: Dispõe sobre as condições para a promoção, proteção e recuperação da saúde, a organização e o funcionamento dos serviços correspondentes e dá outras providências. Diário Oficial da República Federativa do Brasil. Disponível em: <http://www6.senado.gov. $\mathrm{br} /$ sicon/ExecutaPesquisaLegislacao.action >. Acesso em: 20 fev. 2014.

[7] BRASIL. Ministério da Saúde. Índice de Desempenho do SUS (IDSUS). Disponível em: $<$ http://189.28.128.182/i3geo/sage_tabelas/avaliacao/iframe_notas_2011.php>. Acesso em: 25 fev. 2014.

[8] BRASIL. Decreto no 6.800 de 18 de março de 2009. Dá nova redação ao art. $2^{\circ}$ do Decreto no 1.948, de 3 de julho de 1996, que regulamenta a Lei no 8.842, de 4 de janeiro de 1994, que dispõe sobre a Política Nacional do Idoso, e dá outras providências. Diário Oficial da União, Poder Executivo, 19 de mar. 2009. Disponível em: <http://portal.saude.gov.br/portal/arquivos/pdf/2528\%20aprova\%20a\%2opolitica\%2onacional\%2ode\%2osaude\%2oda\%2opessoa\%2oidosa. pdf $>$. Acesso em: 20 fev. 2014.

[9] BRASIL. Ministério da Saúde. Panorâmico. Brasília: Organização Pan-Americana de Saúde, 2009. 60 p. (Painéis indicadores do SUS, 7, v. III).

[10] BRASIL. Ministério da Saúde. Secretaria de Atenção à Saúde. Departamento de Ações Programáticas e Estratégicas. Política Nacional de Atenção Integral à Saúde do Homem - princípios e diretrizes. Ministério da Saúde, Secretaria de Atenção à Saúde, Departamento de Ações Programáticas e Estratégicas, Área Técnica Saúde do Homem. Brasília, 2008. 4op.

[11] BRUCKI, S.M.D. et al. Sugestões para o uso do mini-exame do estado mental no Brasil. Arq. Neuro-Psiquiatr. São Paulo, v.61, n.3B, p. 777-781, mai. 2003. DOI: http://dx.doi.org/10.1590/Sooo4-282X2003000500014

[12] CICONELLI, R.M. et al. Tradução para a língua portuguesa e validação do questionário genérico de avaliação de qualidade de vida SF-36 (Brasil SF-36).

Rev. Bras. Reumatol. Campinas, v.39, n.3, p. 143-150, nov. 1999.

[13] CNS. Conselho Nacional de Saúde. Relatório final da roa Conferência Nacional de Saúde. SUS: construindo um modelo de atenção à saúde para a qualidade de vida. Brasília: Ministério da Saúde; 1996. Disponível em: <http:// conselho.saude.gov.br/biblioteca/Relatorios/relatorio_10.pdf $>$. Acesso em: 8 fev. 2014.

[14] CRUZ, M.S. et al. Prevalência de deficiência auditiva referida e causas atribuídas: um estudo de base populacional. Cad. Saúde Pública, Rio de Janeiro, v. 25, n. 5, 2009. Disponível em: <http://www.scielo.br/scielo.php?script=sci arttext\&pid=So102-311X2009000500019\&lng=pt\&nrm=iso $>$. Acesso em: 4 mar. 2014. DOI: http://dx.doi.org/10.1590/S0102-311X2009000500019

[15] CURIATI, J.A.E.; GARCIA, Y.M. Aspectos propedêuticos. In: CARVALHO FILHO, E.T.; PAPALÉO NETO, M. Geriatria: fundamentos, clínica e terapêutica. 2ed. São Paulo: Atheneu, 2006. p.63-71.

[16] DIAS, A.; CORDEIRO, R.; CORRENTE, J.E. Incômodo causado pelo 
zumbido medido pelo Questionário de Gravidade do Zumbido. Rev. Saude Publica. São Paulo, v. 40, n.4, p. 706-711, ago. 2006. DOI: http://dx.doi. org/10.1590/So034-89102006000500022

[17] FERREIRA, L.M.B.M.; RAMOS JUNIOR, N.A.; MENDES, E.P. Caracterização do zumbido em idosos e de possíveis transtornos relacionados. Rev. Bras. Otorrinolaringol. São Paulo, v.75, n. 2, p. 245-248, mar. 2009. DOI: http:// dx.doi.org/10.1590/Soo34-72992009000200015

[18] FIGUEIREDO. W. Assistência à saúde dos homens: um desafio para os serviços de atenção primária. Cien. Saude Colet. Rio de Janeiro, v.10, n.1, p. 105-109, mar. 2005. DOI: http://dx.doi.org/10.1590/S1413-81232005000100017

[19] GEIB, L.T.C. Determinantes sociais da saúde do idoso. Cien. Saude Colet. Rio de Janeiro, v.17, n.1, p.123-133, jan. 2012. DOI: http://dx.doi.org/10.159o/ S1413-81232012000100015

[20] IBGE. Instituto Brasileiro de Geografia e Estatística. IBGE Cidades, 2013. Disponível em: <http://www.ibge.gov.br/cidadesat/painel/painel. php? codmun=350070\#topo $>$. Acesso em: 12 fev. 2014.

[21] IBGE. Instituto Brasileiro de Geografia e Estatística. Sobre a condição de saúde dos idosos: indicadores selecionados. Rio de Janeiro, 2009. Disponível em: <http://www.ibge.gov.br/home/estatistica/população/indic_sociosaude/2009/com_sobre.pdf>. Acesso em: 12 jan. 2014.

[22] IBGE. Instituto Brasileiro de Geografia e Estatística. Uma análise das condições de vida da população brasileira. Rio de Janeiro: Instituto Brasileiro de Geografia e Estatística (IBGE), 2008.

[23] KNOBEL, K.; ALMEIDA, K. Perfil dos pacientes em terapia para habituação do zumbido (TRT). São Paulo, 2000. Monografia (Especialização em Audiologia Clínica) CEFAC-CEDIAU, São Paulo, 2000.

[24] KONG, K.H.; YANG, S.Y. Health-related quality of life among chronic stroke survivorsattending a rehabilitation clinic. Singapore Med. J. Singapore, v.47, n.3, p. 213-218, dez. 2006.

[25] LANDEIRO, G.M.B. et al. Revisão sistemática dos estudos sobre qualidade de vida indexados na base de dados SciELO. Ciênc. saúde coletiva, Rio de Janeiro, v. 16, n. 10, 2011. Disponível em: <http://www.scielo.br/scielo.php?script=sci arttext\&pid=S1413-81232011001100031\&lng=pt\&nrm=iso $>$. Acesso em: 26 fev. 2014. DOI: http://dx.doi.org/10.1590/S1413-81232011001100031

[26] LEBRÃO, M.L.; LAURENTI, R. Condições de saúde. In: LEBRÃO, M.L.; DUARTE, Y.A.O. SABE: saúde, bem-estar e envelhecimento: o Projeto SABE no município de São Paulo: uma abordagem inicial. Brasília: Organização Pan-Americana da Saúde; 2003. p. 73-91.

[27] LEPRE, R.M.; MARTINS, M.F.A. Desenvolvimento humano e educação: diversidade e inclusão. In: CAPELLINI, V.L.M.F. (Org). Práticas em educação especial e inclusiva na área da deficiência mental. Bauru: $\mathrm{MEC} / \mathrm{FC} / \mathrm{SEE}$, 2008. p.16-20.

[28] LIMA, M.G. et al. Health related quality of life among the elderly: a 
population-based study using SF-36 survey. Cad. Saúde Pública, Rio de Janeiro, v. 25, n. 10, 2009. Disponível em: <http://www.scielo.br/scielo.php?script=sci arttext\&pid=So102-311X2009001000007\&lng=pt\&nrm=iso $>$. Acesso em: 4 mar. 2014. DOI: http://dx.doi.org/10.1590/So102-311X2009001000007

[29] MONDELLI, M.F.C.G.; ROCHA, A.B. Correlação entre os achados audiológicos e incômodo com zumbido. Arq. Int. Otorrinolaringol. v.15, n.2, p. 172180, mai. 2011. DOI: http://dx.doi.org/10.1590/S1809-48722011000200009

[30] PAPALÉO NETO, M.; KLEIN, E.L.; BRITO, F.C. Avaliação Geriátrica Multidimensional. In: CARVALHO FILHO, E.T.; PAPALÉO NETO, M. Geriatria: fundamentos, clínica e terapêutica. 2ed. São Paulo: Atheneu, 2006. p.73-85.

[31] PIMENTA, F.A.P. et al. Avaliação da qualidade de vida de aposentados com a utilização do questionário Sf - 36. Rev Assoc Med Bras. São Paulo, v.54, n.1, p. 55-60, dez. 2008. DOI: http://dx.doi.org/10.1590/So104-42302008000100021

[32] SANCHEZ, T.G. Zumbido: estudo da relação entre limiar tonal e eletrofisiológico e das respostas elétricas do tronco cerebral. 1997. 18op. Tese - Faculdade de Medicina da Universidade de São Paulo, Universidade de São Paulo, São Paulo, 1997.

[33] SANCHEZ, T.G. et al. Controvérsias sobre a Fisiopatologia do Zumbido. Arq. Fund. Otorrinolaringol. São Paulo, v.1, n.1, p. 2-8, jan, 1997.

[34] SOUZA, R.R. Anatomia do Envelhecimento. In: CAVALHO FILHO, E.T.; PAPALÉO NETO, M. Geriatria: fundamentos, clínica e terapêutica. zed. Editora: Atheneu, 2006. p.35-42.

[35] SZWARCWALD, C.L. et al. Socio-demographic determinants of self-rated health in Brazil. Cad. Saúde Pública, Rio de Janeiro, v. 21, supl. 1, 2005. DOI: http://dx.doi.org/10.1590/So102-311X2005000700007

PATRÍCIA RIBEIRO MATTAR DAMIANCE pesquisadora do Grupo de Estudo e Pesquisa sobre o Envelhecimento (GREPEN), docente da Universidade do Sagrado Coração (USC) e doutoranda em Saúde Coletiva da Faculdade de Odontologia de Bauru da Universidade de São Paulo (FOBUSP) - e-mail:patricia.mattar@usp.br

ALINE MEGUMI ARAKAWA pesquisadora do Grupo de Estudo e Pesquisa sobre o Envelhecimento (GREPEN), professora da Coordenadoria Especial de Fonoaudiologia da Universidade Federal de Santa Catarina e doutoranda em Saúde Coletiva da Faculdade de Odontologia de Bauru da Universidade de São Paulo (FOB-USP) - e-mail: arakawaaline@gmail.com

ELEN CAROLINE FRANCO pesquisadora do Grupo de Estudo e Pesquisa sobre o Envelhecimento (GREPEN) e doutoranda em Saúde Coletiva da Faculdade de Odontologia de Bauru da Universidade de São Paulo (FOB-USP) - e-mail: elen.fono@yahoo.com.br

THAÍSA RINO DE FREITAS COELHO mestra em Odontologia em Saúde Coletiva e doutoranda em Saúde Coletiva da Faculdade de Odontologia de Bauru da Universidade de São Paulo (FOB-USP) -e-mail: thaisarino@usp.br 
MARISTELA APARECIDA DOS SANTOS DA SILVA mestra em Saúde Coletiva (FOB-USP) e doutoranda em Saúde Coletiva pela Faculdade de Odontologia de Bauru da Universidade de São Paulo (FOB-USP) - e-mail: marissilva@usp.br

JOSÉ ROBERTO PEREIRA LAURIS doutor em Distúrbio da Comunicação Humana e professor titular do Departamento de Odontopediatria, Ortodontia e Saúde Coletiva da Faculdade de Odontologia de Bauru da Universidade de São Paulo (FOB-USP) - e-mail: jrlauris@fob.usp.br

MAGALI DE LOURDES CALDANA coordenadora do Grupo de Estudo e Pesquisa sobre o Envelhecimento (GREPEN), professora Associada do Curso de Fonoaudiologia e dos Programas de Pós Graduação em Fonoaudiologia e em Ciências Odontológicas Aplicadas da Faculdade de Odontologia de Bauru da Universidade de São Paulo (FOB-USP) - e-mail: mcaldana@fob.usp.br

JOSÉ ROBERTO DE MAGALHÃES BASTOS professor titular da Faculdade de Odontologia de Bauru da Universidade de São Paulo (FOB-USP) e pós-doutorado em Ciências da Saúde da Escola Superior de Medicina Dentária de Lisboa em Portugal-e-mail: zeromaba@fob.usp.br 